\title{
Factores que afectan la sostenibilidad y el crecimiento de las pequeñas empresas en la ciudad de Bucaramanga - Santander - Colombia
}

\author{
Jorge Alberto Bravo Geney \\ Corporación Universitaria de Ciencia y Desarrollo - UNICIENCIA, \\ Bucaramanga, Colombia \\ Universidad de Baja California, México. \\ Correo electrónico autor: jorge.bravo@unicienciabga.edu.co \\ Recepción: 5 Octubre 2017 \\ Aprobación: 5 Diciembre 2017
}

\begin{abstract}
Resumen
La globalización es un concepto que abre las puertas de nuevos mercados y por tanto implica estar preparado para brindar a los clientes externos, las condiciones necesarias para satisfacer sus necesidades más allá de las expectativas que tengan. Esto implica que los objetivos específicos de permanencia y crecimiento se hacen cada vez más difíciles de alcanzar, si no se cuenta con los elementos que garanticen una sana competencia. La innovación y la tecnología, se convierten entonces en ingredientes indispensables para ofrecer productos o servicios de alta calidad, con mínimos costos y en condiciones de eficiencia, eficacia y oportunidad. Para lograr los objetivos propuestos, se trabajó con una muestra representativa de la población de pequeñas empresas de la ciudad de Bucaramanga, con el fin de identificar las necesidades que padecen las empresas de la región en materia financiera que afectan el crecimiento y sostenibilidad de las mismas a largo plazo en los cinco principales sectores económicos, identificados de acuerdo con información suministrada por la Cámara de Comercio de la ciudad.
\end{abstract}

Palabras clave: Competencias financieras, Pymes, sectores económicos, sostenibilidad, generación de valor. 


\title{
Factors affecting the sustainability and growth of small enterprises in the city of Bucaramanga, Santander, Colombia
}

\begin{abstract}
Globalization is a concept that opens the door to new markets and therefore implies being prepared to provide external customers with the necessary conditions to meet their needs beyond the expectations they have. This implies that the specific objectives of permanence and growth become increasingly difficult to reach, without the elements that guarantee healthy competition. Innovation and technology, then, become indispensable ingredients to offer products or services of high quality, with minimum costs and in conditions of efficiency, effectiveness and opportunity. In order to achieve the proposed objectives, a representative sample of the population of small enterprises of the city of Bucaramanga was used to identify the needs of the region's companies in financial matters that affect the growth and sustainability of The same in the long term in the five main economic sectors, identified according to information provided by the city's Chamber of Commerce.
\end{abstract}

Keywords:Financial skills, SMEs, economic sectors, sustainability, value creation.

\section{Introducción}

La globalización es un fenómeno que exige que los países promuevan la educación como alternativa para incentivar y alcanzar el desarrollo y la competitividad. Es por ello que la UNESCO (2016) señala que

Las tecnologías de la información y la comunicación (TIC) pueden contribuir al acceso universal a la educación, la igualdad en la instrucción, el ejercicio de la enseñanza y el aprendizaje de calidad y el desarrollo profesional de los docentes, así como a la gestión dirección y administración más eficientes del sistema educativo (p.37).

Se inició con la identificación de las necesidades del área financiera de las pequeñas empresas de la ciudad de Bucaramanga en sus principales sectores económicos, lo que permitirá, para las Instituciones de educación superior de la región, evaluar las competencias financieras con las que están formando a los futuros egresados para proponer alternativas de solución a los diferentes problemas que las empresas presentan en la ciudad. Lo anterior facilitará el diseño de planes estratégicos que fortalezcan esos procesos educativos, de tal manera que las asignaturas que hacen parte del componente financiero de los diferentes planes de estudio, le aporten al futuro egresado, las competencias necesarias que garanticen un desempeño exitoso en el entorno laboral, que cada vez es más competitivo y globalizado.

El tipo de investigación realizada fue 
descriptiva puesto que el objetivo fue identificar cuáles son los factores que afectan el crecimiento y sostenibilidad de las pequeñas empresas a largo plazo en la ciudad de Bucaramanga, para posteriormente diseñar una matriz DOFA, que evidencia el estado de los factores internos y externos de estas organizaciones.

\section{Estado del arte. El área financiera en las organizaciones}

El objetivo básico financiero de toda organización es maximizar el valor de la empresa y para lograrlo se deben tomar decisiones bajo una perspectiva de largo plazo buscando siempre la permanencia y el crecimiento en el mercado. En el día a día de las empresas, se toman decisiones de inversión, de financiación y reparto de utilidades, para lo cual se debe contar con las habilidades y competencias necesarias, que permitan tomarlas de manera acertada y oportuna.

La globalización y la apertura de nuevos mercados, trajo consigo que el manejo de la información financiera debiera hacerse siguiendo estándares internacionales, de tal manera que se maneje un lenguaje universal. Nacen de esta manera las normas internacionales de información financiera, NIIF, las que buscan que la información financiera sea fiable y refleje la realidad de los negocios, para de esa forma tomar decisiones enfocados al alcance del objetivo básico financiero.

Álvarez y Abreu (2008) sostienen que es muy importante que las pequeñas y medianas empresas tengan estrategias financieras claras que minimicen las condiciones de riesgo y obtener así resultados favorables. Los procesos de planeación financiera permiten a las empresas anticiparse a las diversas situaciones y prepararse ante eventuales circunstancias que pueden afectar la sostenibilidad de la empresa en el largo plazo.

Para la determinación de estrategias financieras se deben de primero que nada identificar las áreas claves en las que nos vamos a enfocar, que en este caso son: el director financiero, la competitividad, la innovación, la financiación y el riesgo financiero, la información financiera y los estados financieros (p. 26).

Según manifiesta Chiavenato, I. (2010) ...cuanto más fuerte sea la tempestad y vigoroso el oleaje del mar, tanto más atento y seguro debe estar el timonel frente a los desafíos de las aguas por donde navega. De igual manera, la turbulencia y la incertidumbre que caracterizan al mundo actual de los negocios incesantemente presentan enormes desafíos a las organizaciones (p. 76).

A medida que transcurre el tiempo y las circunstancias del mercado son más exigentes en cuanto a las condiciones de competitividad, las empresas deben estar preparadas estratégicamente 
para contrarrestar los efectos que tal situación pueda ocasionar en todos los ámbitos de la organización.

Todas las organizaciones compiten por obtener recursos, mercados, clientes, personas, imagen y prestigio. Actúan como agentes dinámicos dentro del contexto variable e incierto que generan los rápidos cambios que sufren las sociedades, los mercados, las tecnologías, el mundo de los negocios y el medio ambiente. Esto exige que los administradores y dirigentes de dichas organizaciones comprendan, por un lado, las dinámicas y las tendencias de sus respectivos sectores de actividad, y por otro lado, formulen estrategias creativas que motiven a las personas y aseguren un desempeño superior con el propósito de garantizar la sustentabilidad de la organización en un mundo de negocios que cambia continuamente.

Chiavenato agrega que estos cambios interminables, llevan a las organizaciones a la renovación y revitalización, y necesariamente a su transformación. Si el contexto se modifica para funcionar, entonces es preciso que la organización, por lo menos, esté atenta a los cambios que se registran a su alrededor para mantenerse actualizada y lista para competir. Es cuestión de supervivencia.

No existe fórmula o patrón para crear una organización exitosa y de alto desempeño. Depende más de la iniciativa que tomen, su voluntad y anticipación a los desafíos que surgen a cada instante, propiciando cambios en vez de adaptarse al entorno, solo por reacción.

\section{Estrategia: Fundamentos}

Kuhn, T. (1972) decía

La diferencia entre el trabajo científico y la del estratega es que el primero se concentra en problemas orientados hacía una disciplina o ciencia específica, mientras que los que afronta el estratega son interdisciplinarios e implican una amplia gama de conocimientos (p. 89).

La estrategia organizacional es el patrón de decisiones que integra los objetivos generales de una organización en un todo coherente y con un propósito. Una estrategia bien formulada permite asignar e integrar todos los recursos y las competencias organizacionales en una proposición única y viable, para anticiparse a los cambios del entorno y a las contingencias frente a competidores preparados que luchan por obtener los mismos clientes y proveedores.

\section{Necesidad de una planeación Estratégica}

La evidencia demuestra que, por lo general, las organizaciones que planean su 
estrategia registran un desempeño superior que afectan su viabilidad, permanencia, a las que no lo hacen. Las que tienen éxito crecimiento y evolución. Sin embargo, si se aplica procuran que su estrategia sea acorde con las de forma incompleta, resultará insuficiente, pues condiciones del entorno. Asimismo, la estrategia no se trata solamente de cubrir las acciones de corto define la estructura y los procesos internos de la plazo. En el proceso de Planeación estratégica es organización con la expectativa de que produzcan preciso formular de forma integrada y articulada efectos muy positivos en su desempeño.

Los principales beneficios de la planeación estratégica son:

- Claridad de la visión estratégica de la organización. todos los planes tácticos y operativos. La figura siguiente muestra este proceso de integración.

\section{Marco Teórico Metodológico. Diseño}

\section{de la investigación}

La presente investigación parte de un estudio cuantitativo y cualitativo, basado

- Comprensión del entorno sumamente cambiante y competitivo.

- Enfoque dirigido mediante objetivos a largo plazo, a lo que tendrá importancia estratégica para la organización en el futuro.

- Comportamiento proactivo frente a los elementos del entorno externo, de modo independiente a los del entorno interno.

- Comportamiento sistémico y holístico que involucre a toda la organización.

- Interdependencia con el entorno externo.

La Planeación estratégica está ligada directamente con los objetivos de la organización en encuestas a una muestra de pequeñas empresas de la ciudad de Bucaramanga, para lo cual se identificaron los cinco principales sectores económicos basados en información suministrada por la Cámara de Comercio de la ciudad.

\section{Tipo y nivel de investigación}

Esta investigación es de tipo descriptivo, puesto que busca especificar las propiedades importantes de personas, grupos, comunidades o cualquier otro fenómeno que sea sometido a análisis. Específicamente se pretendió identificar las necesidades financieras de las pequeñas empresas de Bucaramanga que permitan a las instituciones de educación superior de la región, 
evaluar la coherencia de las competencias financieras de sus egresados en contraste con los requerimientos del entorno. Al tiempo es una investigación de campo, puesto que se basa en informaciones que provienen entre otras fuentes, de entrevistas, cuestionarios, encuestas y observaciones, apoyándose en fuentes de carácter documental.

\section{Población y muestra}

Para la determinación de la población fue necesario en primer lugar, identificar los principales sectores económicos de la ciudad de Bucaramanga, para seguidamente determinar la cantidad de pequeñas empresas registradas en la Cámara de Comercio de esta ciudad en esos sectores con el porcentaje de participación correspondiente, lo que permitió detallar la población total.

En el ranking por sectores económicos de las empresas registradas en la Cámara de Comercio de la ciudad de Bucaramanga se relacionan 21 sectores económicos, que al clasificarlos por el nivel de sus activos, registran los siguientes cinco sectores como los más representativos de la ciudad:

- La construcción.
- Actividades financieras y de seguros.

- Comercio al por mayor y al por menor, reparación de vehículos automotores y motocicletas .

- Agricultura, ganadería y caza.

- Suministro de Electricidad, gas, vapor y aire acondicionado.

De acuerdo al análisis realizado, la ciudad de Bucaramanga tiene un total de 44.512 empresas registradas en la Cámara de comercio de Bucaramanga, información suministrada con corte jueves 13 de octubre de 2016, entre las cuales 41.317 son microempresas, 2.447 corresponden a pequeñas empresas y 603 son medianas, tomado desde su clasificación de acuerdo a su tamaño.

\section{Población}

Está conformada por las pequeñas empresas correspondientes a los cinco principales sectores económicos de la ciudad de Bucaramanga. De acuerdo a la investigación realizada, se comprobó la existencia de 607 pequeñas empresas inscritas en Cámara de Comercio de Bucaramanga, con corte al 13 de octubre de 2016, correspondientes a los cinco sectores económicos principales de la ciudad de 
Bucaramanga.

Por lo anterior, la población está conformada por las mencionadas 607 pequeñas empresas para las que se calculan los porcentajes de participación utilizados para caracterizar la muestra, cuyo valor resultante fue de 86 empresas, lo que se puede apreciar en la tabla 1.

Tabla 1. Muestra de pequeñas empresas por sectores económicos

\begin{tabular}{|l|c|c|}
\hline \multicolumn{1}{|c|}{ Sectores económicos } & $\begin{array}{c}\% \text { de partici- } \\
\text { pación }\end{array}$ & $\begin{array}{c}\text { Cantidad en } \\
\text { la muestra }\end{array}$ \\
\hline $\begin{array}{l}\text { Comercio al por mayor y al por menor, reparación de vehículos } \\
\text { automotores y motocicletas. }\end{array}$ & $56,34 \%$ & 48 \\
\hline Construcción. & $32,78 \%$ & 28 \\
\hline Agricultura, ganadería y caza. & $7,25 \%$ & 6 \\
\hline Actividades financiera y de seguros. & $3,13 \%$ & 3 \\
\hline Suministro de electricidad, gas, vapor y aire acondicionado. & $0,49 \%$ & 0 \\
\hline TOTAL & $100 \%$ & 86 \\
\hline
\end{tabular}

Fuente: Autor del proyecto

\section{Técnicas y métodos}

El método utilizado fue el inductivo, puesto que a partir de verdades particulares se concluyeron verdades generales. Al identificar las necesidades financieras de las pequeñas empresas de la ciudad de Bucaramanga, se contrastaron con las competencias en el área que ofrece la Institución objeto de estudio, actividad que se puede replicar a los diferentes programas académicos de la región, con el objetivo de proponer planes de mejoramiento que fortalezcan los procesos de aprendizaje ante las exigencias del entorno.

\section{Instrumentos}

El instrumento utilizado para la recolección de la información fue la encuesta, que se aplicó a la muestra objeto de estudio, compuesta por las pequeñas empresas de los cinco principales sectores económicos de la ciudad de Bucaramanga. Las variables que se tuvieron en cuenta para la redacción de las preguntas de la encuesta aplicada a las pequeñas empresas, fueron las seis áreas empresariales más representativas: planeación estratégica, gestión administrativa, gestión humana, gestión de calidad, gestión comercial y gestión financiera, teniendo en cuenta que cualquier situación que 
las afecte, impactará en la generación de valor de la organización.

\section{Análisis de resultados}

\section{Diagnóstico basado en análisis de} resultados

Teniendo en cuenta que la encuesta fue diligenciada por el $97 \%$ del total de la muestra, por personal que interviene en la toma de las decisiones financieras de las empresas encuestadas, a continuación se presenta el diagnóstico de las necesidades de las pequeñas empresas registradas en la Cámara de Comercio de la ciudad de Bucaramanga, como resultado del proceso de investigación realizado:

\section{Planeación estratégica}

La dinámica de los negocios hoy día, ha llevado a que las PYMES reaccionen de manera rápida a los cambios del mercado y se organicen de forma estructurada para lograr la sostenibilidad y el crecimiento empresarial. Las exigencias comerciales y de servicios requieren que el empresario trabaje con una planeación estratégica que oriente la empresa a cubrir las necesidades del mercado y poder asumir el reto de enfrentarse a los retos que impone la globalización, a negociar con proveedores y a entablar unas óptimas relaciones con los acreedores.

En este sentido, se observa que muchas pequeñas empresas han tenido que formalizar sus procesos y evolucionar para adaptarse a los cambios que el entorno exige, es así como se evidencia que los procesos como la planeación estratégica retoman importancia en estos entes económicos. Por tanto, el $77 \%$ del personal conoce la misión y están involucrados activamente en el logro de los objetivos empresariales y el $66 \%$ del personal tiene participación en el diseño e implementación de estrategias organizacionales.

Sin embargo, existe un porcentaje del $20 \%$ del personal que no conoce la misión, visión, ni objetivos empresariales y un 30\% del personal que no tiene participación en el diseño e implementación de estrategias organizacionales, lo que pone en riesgo la sostenibilidad a largo plazo en el mercado.

En razón a lo anterior, se vuelve imperativo involucrar la totalidad del personal para que sean partícipes en los diferentes niveles de la planeación estratégica, contar con sus experiencias y conocimientos, lo cual redundará en una óptima utilización de todos los recursos disponibles garantizando así el cumplimiento 
de los indicadores de gestión y objetivos que conlleven a la sostenibilidad a largo plazo del ente económico.

\section{Gestión Administrativa}

El éxito o fracaso de las empresas está condicionado en gran parte a tener una buena administración del negocio, es por esto que los profesionales de hoy han adquirido conciencia de la importancia de un buen equipo de trabajo que permita garantizar el éxito empresarial.

Se observa que la mayoría de las pequeñas empresas están adoptado estructuras organizacionales definidas para garantizar su productividad, eficiencia y calidad en el cumplimiento de su misión y procesos, e intentan mantenerse actualizados sobre leyes y normas en materia, laboral, comercial, tributaria y ambiental.

Sin embargo, el 22\% de las empresas no tienen definidos y documentados sus procesos financieros, comerciales y de operaciones, lo que se considera alto por cuanto la inexistencia de procesos financieros documentados dificulta el control interno y aumenta los riesgos inherentes a los procesos, los cuales muchas veces al materializarse ocasionan pérdidas que afectan la continuidad del negocio.

\section{Gestión Humana}

Es de los aspectos más relevantes de las empresas por cuanto el desarrollo de las competencias del talento humano apoya a toda la estructura administrativa, desde la cual se planean y desarrollan las actividades para cumplir los objetivos.

Como resultado de la investigación, se puede observar como fortaleza, que el $80 \%$, manifiesta que el personal tiene la capacidad de comunicación, gestión y liderazgo, atributos que son determinantes para el buen trabajo en equipo y la posibilidad de establecer mejores y más duraderas relaciones laborales.

En contraste, se resalta como mayor debilidad que corresponde al porcentaje negativo más alto, que el $53 \%$ de las empresas encuestadas no exigen al personal conocimientos sobre Tecnologías de la información y las comunicaciones, siendo esta competencia esencial para la toma de decisiones.

También se observa que el $24 \%$ de los encuestados dieron respuesta negativa a tener una visión medio ambiental definida, desconociendo el papel fundamental que actualmente juega la responsabilidad social con el planeta y las leyes medio ambientales. La sustentabilidad 
en el alcance del éxito y la consolidación de los negocios hace que las empresas requieran profesionales en el área financiera, que tengan competencias para incorporar este tema a sus estrategias.

Por último y no menos importante, se detalla como debilidad con un $24 \%$ de las respuestas negativas, el hecho que las empresas no realizan procesos de inducción y re inducción a sus empleados, y evidencia que en muchas ocasiones se considera que basta con haber seguido un riguroso proceso de reclutamiento y selección de personal para garantizar que el empleado desempeñe sus actividades de manera óptima. Sin embargo, cuando esta persona no cuenta con la información necesaria para saber qué espera la empresa de ella, difícilmente se puede integrar al trabajo y convertirse en un buen elemento para la empresa.

\section{Gestión de calidad}

La gestión de calidad aporta beneficios a la empresa como la planeación de todos sus procesos, que permite estandarizar los diferentes procedimientos, para poder resolver cualquier situación que se presente, adicionalmente permite realizar un seguimiento y mejora a todos los procesos con el fin de optimizar la calidad en los productos y servicios que presta el ente económico.

En razón a lo anterior, y como resultado de las encuestas realizadas se observa con el $84 \%$, que los productos o servicios de la empresa cumplen con las normas técnicas nacionales o internacionales establecidas de acuerdo a cada sector o actividad económica, teniendo en cuenta que existen estándares para implementar a través de un sistema de gestión de la calidad.

En contraste se detalla como debilidad que el $31 \%$ corresponde a las respuestas negativas a la pregunta referida a la capacitación por parte de la empresa a sus colaboradores en temas de calidad y mejoramiento, resaltando nuevamente como debilidad la falta de capacitación al talento humano de las empresas y sobre todo en temas de calidad de vital importancia para:

- Establecer ventajas competitivas en el mercado.

- Generar mayor rentabilidad.

- Aumentar el grado de satisfacción de los clientes internos y externos.

- Permitir que la empresa está siempre atenta a las necesidades del cliente. 


\section{Gestión comercial}

A través de la gestión comercial se canaliza el contacto directo con los clientes externos dando cumplimiento a los objetivos propuestos e indicadores conforme a los presupuestos establecidos. Es por esto, que al realizar el análisis se evidencia como debilidad con un porcentaje negativo de respuesta del $24 \%$, el hecho que las empresas no se encuentren bien posicionadas en el mercado y que no estén realizando análisis de su competencia; a su vez, con un porcentaje negativo de respuesta del $21 \%$, se encuentra que las empresas no tienen políticas de servicio al cliente, fidelización de clientes, postventa y clientes nuevos, lo que puede ocasionar bajas en las ventas, incumplimiento de los presupuestos establecidos y metas de la empresa que pueden afectar la sostenibilidad y crecimiento de la misma.

\section{Gestión Financiera}

Al momento de tomar cualquier decisión de financiamiento e inversión se debe tener en cuenta la información financiera existente para traducirla en estrategias financieras que conlleven al crecimiento y sostenibilidad de las PYMES en el mercado, siendo más competitivas y mejorando su productividad. Esta gestión debe ser organizada y debe estar acoplada a la normatividad vigente.

De acuerdo al análisis realizado se toman como fortalezas en la gestión financiera de las pequeñas empresas de Bucaramanga las siguientes:

La información financiera de las empresas es confiable, oportuna, útil y se usa para la toma de decisiones teniendo en cuenta el porcentaje afirmativo de respuesta del $88 \%$.

La empresa tiene un sistema establecido para contabilizar y controlar eficientemente sus inventarios con un porcentaje afirmativo de respuesta del $88 \%$.

La empresa cumple con los compromisos adquiridos con sus proveedores de bienes y servicios de manera oportuna con un porcentaje afirmativo de respuesta del $92 \%$.

En contraste, se toman como debilidades en la gestión financiera de las pequeñas empresas de Bucaramanga los siguientes hechos:

- Con un porcentaje de respuesta del $15 \%$, las empresas poseen un nivel de 
endeudamiento sobre el que no ejercen un adecuado control y no estudian de forma apropiada las fuentes de financiación.

- Con un porcentaje de respuesta del $17 \%$, las empresas no cuentan con un sistema claro para establecer sus costos, dependiendo de los productos, servicios y procesos.

\section{Matriz DOFA para las pequeñas empresas de Bucaramanga.}

\section{Factores Internos}

\section{Debilidades}

— El personal que no tiene participación en el diseño e implementación de estrategias organizacionales

- Las empresas NO tienen definidos y documentados sus procesos financieros, comerciales y de operaciones

— Desactualización en políticas de gestión humana

— Bajas en las ventas

— Deficiente actualización en procesos de producción

— Estancamiento de productos
- Falta de aplicación de nuevas tecnologías

— Falta de compromiso de los empleados

— Falta de estrategias de mercado

- Las pequeñas empresas no exigen al personal conocimientos sobre Tecnologías de la información y las comunicaciones

— Falta de visión medio ambiental definida

- No se realizan procesos de inducción y re inducción para empleados antiguos

— Falta de capacitación a sus empleados en temas de calidad y mejoramiento continuo

- Falta de capacitación por parte de la empresa a sus colaboradores en temas de calidad y mejoramiento

— Las empresas no tienen políticas de servicio al cliente, fidelización de clientes, postventa y cliente nuevos

- La empresa no posee un nivel de endeudamiento controlado

— La empresa no cuenta con un sistema claro para establecer sus costos, dependiendo de los productos, servicios y procesos. 


\section{Fortalezas}

— Formalización de sus procesos

- Optima utilización de todos los recursos disponibles, mejora de los procesos y herramientas de trabajo

- Implementación de la planeación estratégica

- Estructuras organizacionales definidas por que es fácil recopilar todos los procesos en las pequeñas empresas

- Actualización permanente en leyes y normas en materia, laboral, comercial, tributaria y ambiental

- Buena capacidad de comunicación personal que permite establecer mejores y más duraderas relaciones laborales

— La información financiera de las empresas es confiable, oportuna, útil y se usa para la toma de decisiones

— La empresa tiene un sistema establecido para contabilizar, controlar y rotar eficientemente sus inventarios

— La empresa cumple con los compromisos adquiridos con sus proveedores de bienes y servicios de manera oportuna.

\section{Factores Externos}

\section{Oportunidades}

— Adaptación a los cambios del entorno

— El precio del dólar es una oportunidad para los exportadores

- El uso de las nuevas tecnologías da acceso a nuevos mercados

- Los productos o servicios de la empresa cumplen con las normas técnicas nacionales o internacionales establecidas de acuerdo a cada sector o actividad económica

— Adopción de las NIIF.

\section{Amenazas}

- Alta carga impositiva

- Alza del dólar hace más costosas algunas materias importadas

- Aumento de la Competencia

- Contrabando

— Falta de competitividad en el mercado

— Situación económica del país (inflación) 
— Vías de comunicación en mal estado

- Las empresas no se encuentren bien posicionadas en el mercado

— Las empresas no realizan análisis de su competencia.

\section{Conclusiones}

Al realizar el análisis del entorno compuesto por las pequeñas empresas de la ciudad de Bucaramanga se concluye que:

- Son pocas las empresas que involucran a sus empleados en la implementación de estrategias organizacionales.

- Las empresas solo involucran a sus empleados en la misión, visión y cumplimiento de los objetivos empresariales.

- La mayoría de las empresas han formalizado, definido y documentado sus procesos comerciales, financieros y operativos y se encuentran actualizadas en materia ambiental para la implementación de estrategias operativas.

- Las empresas se actualizan en materia laboral, financiera, tributaria y ambiental.

- El conocimiento de las Tecnologías de la Información y la Comunicación, TIC, aun no es un requisito indispensable en las empresas como competencia esencial para la toma de decisiones.

- El personal de las empresas está capacitado para liderar y establecer relaciones laborales duraderas y equipos de trabajo eficientes.

- Las empresas permanentemente actualizan y capacitan al personal nuevo $\mathrm{y}$ antiguo.

- Las principales amenazas de las empresas se dan por la inconstante economía del país, por el aumento en la competencia y la fijación del precio del dólar para aquellas que tienen relaciones con organizaciones del exterior.

\section{Recomendaciones}

- Mejorar la infraestructura tecnológica de las instituciones educativas, de tal manera que se garantice a los estudiantes contar los elementos necesarios para desarrollar las competencias propuestas en los diferentes cursos del programa académico que desarrolla.

- Promover estrategias pedagógicas por 
parte de los docentes del programa donde las TIC se conviertan en apoyo fundamental para fortalecer las competencias propuestas.

- Motivar a los docentes de los programas académicos para el diseño de aulas extendidas virtuales, donde se pueda complementar la formación académica de los estudiantes.

- Reestructurar el portafolio de electivas en los planes de estudio, con el fin de implementar asignaturas enfocadas en las TIC en sus diferentes niveles, de tal manera que el estudiante se fortalezca en su uso y las aplique en la solución de diferentes problemáticas del entorno.

- Motivar y concientizar al estudiante de la importancia de fortalecer su proceso de formación, para convertirse en un profesional competitivo para enfrentar las exigencias del entorno laboral.

\section{Referencias bibliográficas}

Afanador,E.(2012).Aplicación de las tecnologías de la información y la comunicación (TIC) en el proceso de exportación orientado a las pequeñas y medianas empresas (PYMES) de Bucaramanga. Recuperado en: http://hemeroteca.unad.edu.co/index. $\mathrm{php/revista-estrategica-organizacio/}$ article/view/1206. (31/03/2017)

Álvarez, M. (2008). Estrategias financieras en la pequeña y mediana empresa. Recuperado en: http://www.spentamexico.org/v3n2/3(2)\%2065-104.pdf. (18/06/2016)

Bancoldex. (2017). Clasificación de empresas en Colombia. Recuperado en: https://www. bancoldex.com/Sobre-microempresas/ Clasificacion-de-empresas-enColombia315.aspx. (15/04/2017)

Boude, O. (2011). Desarrollo de competencias a través de un ambiente de aprendizaje mediado por TIC en educación superior. Recuperado en: http://scielo.sld.cu/ scielo.php?script $=$ sci12011000300007. $(16 / 06 / 2016)$

Brown, J. (2005). Incorporación de las tecnologías de información y comunicación en la docencia universitaria estatal costarricense: problemas y soluciones. Recuperado en: http://revista.inie.ucr.ac.cr/index.php/aie/ article/viewFile/120/119. (18/06/2016)

CCB. (2016). Base de datos Cámara de Comercio de Bucaramanga. Colombia.

Chiavenato, I. (2001). Administración: teoría, proceso y práctica. Colombia: Editorial Mac Graw Hill.

Cabero, J. (2005). Reflexiones sobre la brecha digital y la educación. Recuperado en: http://sid.usal.es/idocs/F8/FDO22178/ reflexiones.pdf. (18/06/2016)

Cobo, J. (2009). El concepto de tecnologías de la información. Benchmarking sobre las definiciones de las TIC en la sociedad del conocimiento. Recuperado en: http:// www.ehu.eus/ojs/index.php/Zer/article/ view/2636/2184. (03/06/2016)

Chacón, O. (2007). Teoría del Trade-off para la definición de la estructura de financiación de las Pymes de Bucaramanga. Recuperado en: http://repvie.uis.edu.co/ 
index.php/revistauisingenierias/article/ view/402/668. (31/03/2017)

Chinchilla, M. (2014). Diagnóstico de la estructura financiera de las pymes del sector de confecciones del área metropolitana de Bucaramanga. Recuperado en: http://tangara.uis.edu. co/biblioweb/tesis/2014/154052.pdf. $(01 / 04 / 2017)$

Díaz, A. (2011). Competencias en educación. Corrientes de pensamiento $e$ implicaciones para el currículo y el trabajo en el aula. Recuperado en: http://www.7-28722011000300001. $(15 / 04 / 2017)$

Dinero. (2016). Pymes contribuyen con más del $80 \%$ del empleo en Colombia. Recuperado en: http://www.dinero. com/edicion-impresa/caratula/articulo/ porcentaje-y-contribucion-de-las-pymesen-colombia/231854. (31/03/2017)

El Tiempo. (2014). La buena hora de la economía de Bucaramanga. Recuperado en: http:// www.eltiempo.com/archivo/documento/ CMS-13733918. (15/04/2017)

Escobar, G. (2009). Modelo para medir la situación financiera en empresas Pyme. Recuperado en: http://repositorio. utp.edu.co/dspace/bitstream/ handle/11059/581/modelo\%20para $\% 20$ medir.pdf? sequence $=3 \&$ is Allowed $=y$. $(01 / 04 / 2017)$

Gómez, O. (2011). Los costos y procesos de producción, opción estratégica de productividad y competitividad en la industria de confecciones infantiles de Bucaramanga. Recuperado en: http://www.scielo. org.co/scielo.php? pid=S 0120 $81602011000100014 \& \mathrm{script}=\mathrm{sci}$ arttext\&tlng=es. (15/03/2017).

Ianni, O. (1996). Teorías de la globalización. Siglo XXI, Venezuela.
Kuhn, T. (1972). Sociology of Science: selected readings. Penguin Books, Chicago, 1972.

Lugo, M. (2010). Las políticas TIC en la educación de América Latina. Tendencias y experiencias. Recuperado en: https://idus.us.es/xmlui/bitstream/ handle/ $11441 / 32395 / \mathrm{L}$ as \% 20 politicas $\% 20$ tic $\% 20$ en $\% 201 a \% 20$ educacion $\% 20$ de $\% 20$ America $\% 20$ latina.pdf? sequence $=1 \&$ is Allowed $=y$. (17/06/2016).

Marulanda, C. (2013). La gestión del conocimiento en las pymes de Colombia. Recuperado en: http://revistavirtual.ucn. edu.co/index.php/RevistaUCN/article/ view/411/834. (31/03/2017).

Milesi, D. (2007). Desarrollo de ventajas competitivas: Pymes. Recuperado en: https://www.researchgate.net/profile/ Gabriel_Yoguel2/publication/28183461_ Desarrollo_de_ventajas_competitivas_ pymes_exportadoras_exitosas en_Argentina_Chile_y_Colombia/ links $/ 558 \mathrm{a} 9 \mathrm{~b} 9 \mathrm{~b} 08 \mathrm{ae} 50 \mathrm{~d} 602 \mathrm{c} 8 \mathrm{e} 0 \mathrm{bb} . p d f$. (01/04/2017).

Mutis, S. (2010). Identificación y análisis de estrategias para el mejoramiento de los procesos de manufactura en las pymes del sector metalmecánico de Bucaramanga y su área metropolitana. Recuperado en: http://tangara.uis.edu.co/biblioweb/ tesis/2010/136221.pdf. (15/03/2017).

Pérez, R. (2003). Propuesta de un modelo de gestión humana y cultura organizacional para pymes innovadoras. Recuperado en: http://journal.ean.edu.co/index. $\mathrm{php/Revista/article/viewArticle/233.}$ (01/04/2017).

Ramírez, C. y otros. (2012). Identificación de las principales variables, que en común afectan negativamente el crecimiento y la rentabilidad de las micro y pequeñas empresas del sector 
calzado en el área metropolitana de Bucaramanga. Recuperado en: http://intellectum.unisabana.edu. co/bitstream/handle/10818/6471/ C A R L O S \% 20 A U G U S T O \% 20 RAMIREZ\%20\%28T\%29FINAL. pdf? sequence $=3 \&$ is A 11 owed $=y$. $(01 / 04 / 2017)$.

Salinas, J. (2004). Innovación docente y uso de las TIC en la enseñanza universitaria. Recuperado en: https://www.uoc. $\mathrm{edu} / \mathrm{rusc} / \mathrm{dt} / \mathrm{esp} / \mathrm{salinas} 1104 . \mathrm{pdf}$. (04/06/2016).

Sánchez, J. (2007). Algunas aproximaciones al problema de financiamiento de las pymes en Colombia. Recuperado en: https://dialnet.unirioja.es/descarga/ articulo/4808086.pdf. (31/03/2017).

UAB. (2003). Las grandes aportaciones de las TIC. Recuperado en: http:// ardilladigital.com/DOCUMENTOS/ TECNOLOGIA\%20EDUCATIVA/ TICs/T 1\%20SOC.INFORMACION/ L A S \% 20 T IC \% 20 Y \% 20 S US \% 20 APORTACIONES $\% 20 \mathrm{~A} \% 20 \mathrm{LA} \% 20$ SOCIEDAD.htm. (04/06/2016).

UNESCO. (2016). Las tecnologías de la información y la comunicación, TIC en la educación. Recuperado en: http://www. unesco.org/new/es/unesco/themes/icts/. $(03 / 06 / 2016)$.

Uniciencia. (2016). Perfiles de formación en Contaduría Pública. Recuperado en: http://www.unicienciabga.edu.co/ programas/contaduria. (04/12/2016).

Valencia, M. (2013). Factores que influyen en el ciclo de vida de las Mi pymes en Bucaramanga y su área metropolitana. Recuperado en: https://revistas.ucc.edu. co/index.php/es/article/view/683/665. $(05 / 03 / 2017)$.
Valenti, P. (2002). La sociedad de la información en América Latina y el Caribe: Tics y un nuevo marco institucional. Recuperado en: $\quad$ http://www.oei.es/revistactsi/ numero2/valenti.htm. (17/06/2016).

Vanguardia. (2012). ¿Por qué mueren tan pronto las empresas en Bucaramanga? Recuperado en: http://www.vanguardia. com/economia/local/143083-por-quemueren-tan-pronto-las-empresas-debucaramanga. (31/03/2017).

Villatoro, P. y Silva, A. (2005). Estrategias, programas $y$ experiencias de superación de la brecha digital y universalización del acceso a las nuevas tecnologías de información y comunicación TIC. Un panorama regional. Recuperado en: https://books.google.es/ books?hl=es\&lr=lang_es\&id=yNL_1 TtQ5XgC\&oi=fnd\&pg=PA5\&dq=Dise $\%$ $\mathrm{C} 3 \% \mathrm{~B} 1 \mathrm{o}+\mathrm{de}+$ un + plan + estrat $\% \mathrm{C} 3 \% \mathrm{~A}-$ 9 gico+para+la+incorporaci $\% \mathrm{C} 3 \% \mathrm{~B}$ $3 n+d e+\operatorname{las}+$ tecnolog $\% \mathrm{C} 3 \% \mathrm{ADas}+-$ $\mathrm{de}+\mathrm{la}+$ informaci $\% \mathrm{C} 3 \% \mathrm{~B} 3 \mathrm{n}+\mathrm{y}+\mathrm{las}+\mathrm{co}-$ municaciones\&ots $=v E Q g i z t N z S \& s i-$

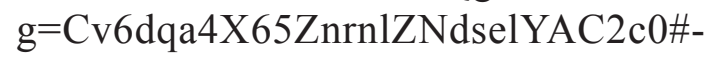
$\mathrm{v}=$ onepage $\& \mathrm{q}=$ Dise $\% \mathrm{C} 3 \% \mathrm{~B} 1 \mathrm{o} \% 20$ de $\% 20$ un $\% 20$ plan $\% 20$ estrat $\%$ C3\%A9 gico $\% 20$ para $\% 201$ a $\% 20$ incorporaci\%C3\%B3n\%20de\%201as\%20tecnolog\%C3\%ADas\%20de \%201a\%20 informaci $\% \mathrm{C} 3 \% \mathrm{~B} 3 \mathrm{n} \% 20 \mathrm{y} \% 201 \mathrm{as} \% 20$ comunicaciones \&f $=$ false. $(17 / 06 / 2016)$.

Zevallos, E. y otros. (2003). Micro, pequeñas $y$ medianas empresas en América Latina. Recuperado en: http:// repositorio.cepal.org/bitstream/ handle/11362/10874/079053070_ es.pdf? sequence $=1 \&$ is Allowed $=y$. (20/03/2017). 